\title{
The Effects of Exogenous Amino Acids on Growth and Nitrogenase Activity in the Cyanobacterium Anabaena cylindrica PCC 7122
}

\author{
By DA VID M. RAWSON \\ Applied Microbiology Group, Department of Science, Luton College of Higher Education, \\ Park Square, Luton LUI 3JU, Bedfordshire, UK
}

(Received 14 March 1985; revised 3 June 1985)

\begin{abstract}
Growth and nitrogenase activity were followed in batch cultures of Anabaena cylindrica PCC 7122 supplemented with a range of protein amino acids and urea at concentrations of 1 and $10 \mathrm{~mm}$. Alanine, arginine, asparagine, aspartate, glutamate, glutamine and serine caused reductions in nitrogenase activity and showed evidence of acting as sources of nitrogen. Suppression of nitrogenase by other amino acids was accompanied by reduced growth. A number of amino acids appeared to be toxic at high concentrations. Cysteine and histidine were toxic at low concentrations.
\end{abstract}

\section{INTRODUCTION}

Cyanobacterial nitrogen metabolism has been widely investigated with regard to nitrogen fixation (Allen \& Arnon, 1955; Gallon, 1980) and the assimilation of inorganic nitrogen sources (Haystead et al., 1973; Batt \& Brown, 1974; Rowell et al., 1977). Amino acid uptake has been noted in several studies (Lee-Kaden \& Simonis, 1982; Thomas et al., 1982) and feed-back inhibition by amino acids of enzymes involved in $\mathrm{NH}_{4}^{+}$assimilation has been reported (Rowell et al., 1977; Stacey et al., 1979).

Studies of the ability of cyanobacteria to utilize amino acids as sole nitrogen sources (Neilson \& Larsson, 1980; Vashampayan, 1982) show highly variable results from different species, and an apparent poor ability amongst photoautotrophs (Neilson \& Doudoroff, 1973). Previous workers have used low amino acid concentrations, usually below $1 \mathrm{~mm}$, and only occasionally have reported on the effect of amino acids on nitrogenase activity.

Possibly the identification of an interrupted tricarboxylic acid cycle in cyanobacteria (Pearce et al., 1969) and the predominance of photoautotrophy have dissuaded surveys of amino acid utilization of the type commonly made of other bacteria and eukaryotic algae. The need for a systematic study of single amino acid utilization and investigation of reported toxic effects was recognized by Stanier \& Cohen-Bazire (1977).

This paper reports the results of a survey of the effects of protein amino acids and urea at two concentrations on the growth and nitrogenase activity of the obligate photoautotroph Anabaena cylindrica PCC 7122 grown in batch culture under continuous illumination.

\section{METHODS}

Organism and culture conditions. Anabaena cylindrica (PCC 7122), obtained from the Pasteur Culture Collection, Paris, France (Rippka et al., 1979), was maintained on agar plates made from BG-1 $1_{0}$ medium (nitrate-free BG-11 medium after Rippka et al., 1979) supplemented with $1.5 \%(\mathrm{w} / \mathrm{v})$ bacteriological agar. Liquid starter cultures were prepared by transfer of inoculum from plate cultures to 50 or $100 \mathrm{ml}$ sterile BG-1 $1_{0}$ medium in conical flasks. Such cultures were grown to late exponential phase $\left(\mathrm{OD}_{663} 1 \cdot 5-1 \cdot 8\right)$, when a single flask culture was used to inoculate all flasks in an experimental run. Investigations of amino acid treated and control cultures were made from cultures grown in $50 \mathrm{ml} \mathrm{BG}-11_{0}$ medium in $250 \mathrm{ml}$ conical flasks under axenic conditions. All amino acid, urea, $\mathrm{NH}_{4} \mathrm{Cl}$ and buffer additions were filter-sterilized. All liquid cultures were maintained at $25^{\circ} \mathrm{C}$, with $5.0 \mathrm{~W} \mathrm{~m}^{-2}$ illumination 
from warm white fluorescent tubes, at 100 oscillations $\mathrm{min}^{-1}$ in an orbital shaker. The effect of amino acids was assayed under both buffered and unbuffered conditions; buffered cultures were kept at pH 7.3 with 5 mM-HEPES, a concentration which kept the $\mathrm{pH}$ constant and which has been shown to have little effect on cell structure and nitrogenase activity (Smith et al., 1983).

Controls, not supplemented with amino acids or urea, but under otherwise identical conditions, were run with each experimental batch and all results are compared with the appropriate control cultures.

Checks for culture purity. The axenic nature of all cultures was checked by plating onto tryptone soya sugar agar, brain heart infusion agar, and BG-11 $1_{0}$ agar, and incubating for $5 \mathrm{~d}$ at $25^{\circ} \mathrm{C}$. Visual checks by phase contrast microscopy were also made.

Measurement of growth. This was followed turbidimetrically at $663 \mathrm{~nm}$ using a $1 \mathrm{~cm}$ path length on $1 \mathrm{ml}$ samples following nitrogenase assay.

Nitrogenase assay. The acetylene reduction method of Stewart et al. (1967) was used.

Chemicals. The purest grade of commercially available chemicals was used throughout. Amino acids were from Sigma, all other compounds were from $\mathrm{BDH}$.

\section{RESULTS}

In Anabaena cylindrica glutamine synthetase has been shown to be the main route of $\mathrm{NH}_{4}^{+}$ assimilation (Rowell et al., 1977). When nitrogen-fixing cultures were supplemented with $\mathrm{NH}_{4}^{+}$ there was a rapid decline in nitrogenase activity accompanied by an increase in the growth rate (Fig. 1 a). This rapid inhibition of nitrogenase is still not fully understood but $\mathrm{NH}_{4}^{+}$is not the sole inhibitor and Stewart (1980) suggests that glutamine synthetase and/or its product is involved.

Urea elicited the same response as $\mathrm{NH}_{4}^{+}$(Figs $1 b$ and $1 c$ ), with an extended period of nitrogenase suppression, confirming the opinion of Neilson \& Larsson (1980) that both atoms of $\mathrm{N}$ are utilized. Whilst statistical analysis showed the growth rate of $\mathrm{NH}_{4} \mathrm{Cl}$-supplemented cultures to be significantly $(P<0.001)$ higher than in the control over the $8 \mathrm{~d}$ period, urea only caused such an effect over the first $5 \mathrm{~d}$ of culture growth. Only minor variations were seen in the length of the lag period.

Unsupplemented BG-1 $11_{0}$-grown cultures had a peak of nitrogenase activity between 45 and $60 \mathrm{~h}$, and reached an $\mathrm{OD}_{663}$ of approximately 2.0 within $192 \mathrm{~h}$. Unbuffered control cultures increased in $\mathrm{pH}$, reaching $9 \cdot 0 \pm 0.2$ by $192 \mathrm{~h}$, and had a slightly higher growth rate than cultures buffered at $\mathrm{pH} 7 \cdot 3$. Additions of amino acids to unbuffered cultures resulted in a range of $\mathrm{pH}$ values as measured at day 8 of the experimental run; the majority of amino acids had a buffering effect. Highest $\mathrm{pH}$ values were found in cultures supplemented with $10 \mathrm{~mm}$ concentrations of arginine $(9 \cdot 2-9 \cdot 4)$, glutamine $(8 \cdot 3-8 \cdot 7)$, proline $(8 \cdot 2-8 \cdot 3)$ and alanine $(7 \cdot 8-8 \cdot 0)$. All other amino acid supplements gave $\mathrm{pH}$ values between $7 \cdot 0$ and $7 \cdot 5$, with the exception of cysteine $(6 \cdot 5-6 \cdot 6)$. This variation in $\mathrm{pH}$ between unbuffered control and amino acid supplemented cultures makes comparison difficult, and unless otherwise stated the results described here refer to buffered conditions.

The patterns of nitrogenase activity and growth in buffered and unbuffered cultures with and without supplements of urea and $\mathrm{NH}_{4}^{+}$were used as points of reference when considering the effect of exogenous protein amino acids on cultures of $A$. cylindrica.

A reduction in nitrogenase activity accompanied by growth equivalent to or above control growth, as seen in cultures supplemented with $\mathrm{NH}_{4}^{+}$and urea, was taken as evidence that the amino acids were acting as a nitrogen source.

Table 1 summarizes the results obtained with 20 amino acids and urea on growth and nitrogenase activity in $A$. cylindrica.

No amino acids reduced nitrogenase activity whilst maintaining growth at or above control levels to the extent shown by $\mathrm{NH}_{4}^{+}$and urea. However, cultures supplemented with arginine, aspartate, glutamine, glutamate (Fig. 2) and serine all had lower nitrogenase activities whilst culture growth was comparable to that of the control. This indicates that these amino acids were able to satisfy in part the nitrogen requirements of $A$. cylindrica. In the case of arginine this effect was most marked at $1 \mathrm{mM}$, the reduction in nitrogenase activity being less marked at $10 \mathrm{mM}$, when the growth rate fell below that of the control over the second half of the experimental period. 


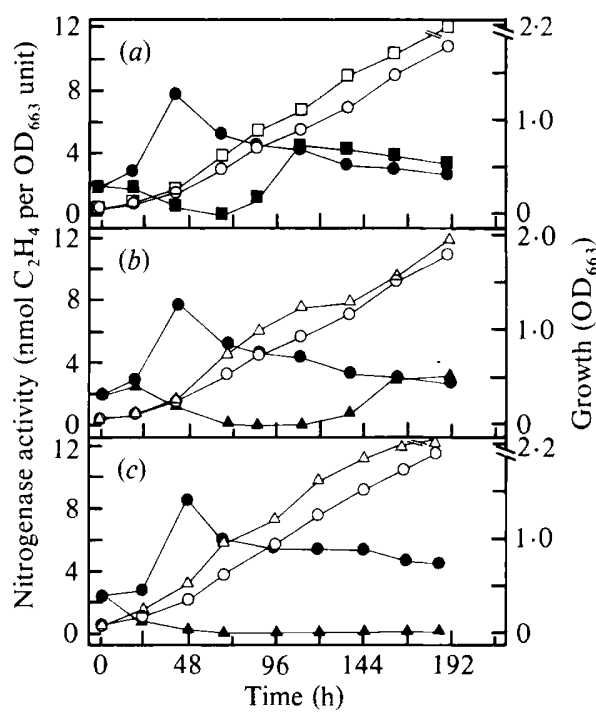

Fig. 1

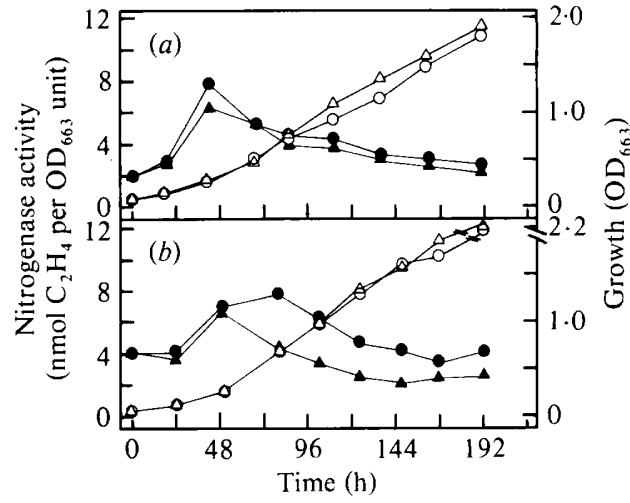

Fig. 2

Fig. 1. Nitrogenase activity $(\square, \Delta, \bigcirc)$ and growth $(\square, \triangle, O)$ of Anabaena cylindrica in control cultures $(\boldsymbol{O}, \bigcirc)$ and in cultures supplemented with $(a) 1 \mathrm{~mm}-\mathrm{NH}_{4} \mathrm{Cl}(\boldsymbol{\nabla}, \square),(b) 1 \mathrm{~mm}$-urea $(\boldsymbol{\Delta}, \triangle)$ and (c) 10 mM-urea $(\boldsymbol{\Lambda}, \triangle)$. All cultures were buffered at $\mathrm{pH} 7 \cdot 3$ with HEPES.

Fig. 2. Nitrogenase activity $(\boldsymbol{\Lambda}, \boldsymbol{)})$ and growth $(\triangle, \bigcirc)$ of Anabaena cylindrica in control cultures $(\boldsymbol{O}$. $\bigcirc)$ and in cultures supplemented with $(a) 1$ mM-glutamate $(\boldsymbol{\Delta}, \triangle)$ and $(b) 10$ mM-glutamate $(\boldsymbol{\Lambda}, \triangle)$. All cultures were buffered at $\mathrm{pH} 7.3$ with HEPES.

Asparagine, glutamine, glutamate and serine caused more pronounced effects at $10 \mathrm{~mm}$. However, whilst there was little reduction in nitrogenase activity in cultures supplemented with $1 \mathrm{~mm}$-glutamate, there was a statistically significant increase in growth rate above that of the control. Similar effects were obtained with both buffered and unbuffered cultures. Little effect was noted with alanine at $1 \mathrm{~mm}$, whilst at $10 \mathrm{~mm}$ buffered cultures had a lower nitrogenase activity and growth rate than the control; unbuffered cultures had a growth rate similar to that of the control but a lower nitrogenase activity.

Asparagine and glutamine are amides which show considerable dissociation into aspartate/glutamate and $\mathrm{NH}_{4}^{+}$. In these cases it was not possible to distinguish between the suppression of nitrogenase caused by the amide or the $\mathrm{NH}_{4}^{+}$dissociation product. The results obtained with glutamate are interesting when compared with those noted by Chapman \& Meeks (1983) with Anabaena variabilis where an exogenous level of $0.5 \mathrm{mM}$-glutamate inhibited growth.

A number of amino acids caused a reduction of nitrogenase activity accompanied by reduced growth. Cultures supplemented with isoleucine, phenylalanine and valine at $1 \mathrm{~mm}$ were not significantly affected but had a pronounced depression of nitrogenase activity and growth at amino acid concentrations of $10 \mathrm{~mm}$.

Leucine, glycine and lysine at $1 \mathrm{~mm}$ and $10 \mathrm{~mm}$ caused a reduction of nitrogenase activity, as did tyrosine at $1 \mathrm{~mm}$ and $2 \mathrm{~mm}$, but this was only accompanied by a major depression in growth at the higher concentrations (Fig. 3), suggesting possible utilization as nitrogen sources at the lower concentrations.

Proline-supplemented cultures showed little sign of perturbation during the first $100 \mathrm{~h}$ of culture, but by $190 \mathrm{~h}$ nitrogenase activity fell to zero at a proline concentration of $10 \mathrm{~mm}$, and this was accompanied by changes in filament morphology as the cells became spherical and filament length was reduced to short 5-20 cell chains.

Threonine, methionine and tryptophan suppressed nitrogenase and prevented growth at a concentration of $10 \mathrm{~mm}$, whilst with methionine and tryptophan at $1 \mathrm{~mm}$ a reduced nitrogenase 
Table 1. Effect of amino acids and urea on growth and nitrogenase activity of Anabaena cylindrica

Results are from $50 \mathrm{ml}$ batch cultures with 5 mM-HEPES buffer at pH 7.3 and the compounds indicated. The percentages are the means of at least three observations.

\begin{tabular}{|c|c|c|c|c|c|c|c|c|c|}
\hline \multirow[b]{2}{*}{ Addition } & \multirow{2}{*}{$\begin{array}{l}\text { Concn } \\
(\mathrm{mM})\end{array}$} & \multicolumn{4}{|c|}{$\begin{array}{c}\mathrm{OD}_{663} \\
\text { (percentage of control) }\end{array}$} & \multicolumn{4}{|c|}{$\begin{array}{l}\text { Nitrogenase activity } \\
\text { (percentage of control) }\end{array}$} \\
\hline & & Day... & 4 & 6 & 8 & 2 & 4 & 6 & 8 \\
\hline \multirow[t]{2}{*}{ L-Alanine } & 1 & 103 & 100 & 100 & 86 & 79 & 81 & 115 & 77 \\
\hline & 10 & 85 & 70 & 69 & 77 & 78 & 85 & 86 & 90 \\
\hline \multirow[t]{2}{*}{ L-Arginine } & 1 & 92 & 123 & 101 & 104 & 102 & 80 & 56 & 0 \\
\hline & 10 & 121 & 101 & 77 & 74 & 128 & 93 & 84 & 69 \\
\hline \multirow[t]{2}{*}{ L-Asparagine } & 1 & 103 & 95 & 102 & 96 & 91 & 85 & 98 & 85 \\
\hline & 10 & 88 & 94 & 94 & 96 & 58 & 80 & 40 & 42 \\
\hline \multirow{2}{*}{ L-Aspartate } & 1 & 88 & 96 & 103 & 103 & 78 & 87 & 70 & 70 \\
\hline & 10 & 108 & 84 & 81 & 86 & 108 & 62 & 69 & 69 \\
\hline \multirow[t]{2}{*}{ L-Cysteine } & 1 & 40 & 15 & 19 & 20 & 0 & 0 & 1 & 17 \\
\hline & 10 & 50 & 13 & 5 & 2 & 0 & 0 & 0 & 0 \\
\hline \multirow[t]{2}{*}{ Glycine } & 1 & 96 & 94 & 88 & 82 & 90 & 86 & 84 & 68 \\
\hline & 10 & 53 & 38 & 26 & 34 & 45 & 34 & 35 & 35 \\
\hline \multirow[t]{2}{*}{ L-Glutamate } & 1 & 104 & 106 & 115 & 106 & 81 & 84 & 89 & 80 \\
\hline & 10 & 108 & 102 & 98 & 108 & 95 & 55 & 52 & 63 \\
\hline \multirow{2}{*}{ L-Glutamine } & 1 & 100 & 99 & 91 & 93 & 98 & 100 & 97 & 74 \\
\hline & 10 & 118 & 97 & 80 & 79 & 93 & 41 & 14 & 0 \\
\hline \multirow[t]{2}{*}{ L-Histidine } & 1 & 62 & 38 & 12 & 10 & 10 & 0 & 0 & 0 \\
\hline & 10 & 46 & 5 & 3 & 1 & 10 & 0 & 0 & 0 \\
\hline \multirow[t]{2}{*}{ L-Isoleucine } & 1 & 100 & 95 & 104 & 82 & 99 & 103 & 108 & 86 \\
\hline & 10 & 76 & 78 & 81 & 78 & 39 & 98 & 66 & 61 \\
\hline \multirow[t]{2}{*}{ L-Leucine } & 1 & 104 & 115 & 76 & 82 & 47 & 21 & 74 & 104 \\
\hline & 10 & 80 & 45 & 31 & 45 & 16 & 27 & 63 & 84 \\
\hline \multirow[t]{2}{*}{ L-Lysine } & 1 & 92 & 90 & 94 & 83 & 108 & 100 & 86 & 22 \\
\hline & 10 & 92 & 71 & 62 & 60 & 90 & 90 & 63 & 0 \\
\hline \multirow{2}{*}{ L-Methionine } & 1 & 86 & 88 & 87 & 73 & 42 & 81 & 110 & 86 \\
\hline & 10 & 63 & 20 & 19 & 20 & 3 & 3 & 10 & 15 \\
\hline \multirow[t]{2}{*}{ L-Proline } & 1 & 107 & 112 & 104 & 83 & 105 & 95 & 68 & 60 \\
\hline & 10 & 87 & 101 & 94 & 89 & 105 & 108 & 47 & 0 \\
\hline \multirow[t]{2}{*}{ L-Phenylalanine } & 1 & 114 & 94 & 91 & 96 & 80 & 98 & 117 & 91 \\
\hline & 10 & 123 & 88 & 77 & 88 & 48 & 65 & 83 & 97 \\
\hline \multirow[t]{2}{*}{ L-Serine } & 1 & 93 & 92 & 91 & 90 & 102 & 95 & 112 & 90 \\
\hline & 10 & 92 & 103 & 100 & 99 & 82 & 92 & 43 & 42 \\
\hline \multirow[t]{2}{*}{ L-Threonine } & I & 92 & 97 & 100 & 100 & 91 & 92 & 35 & 88 \\
\hline & 10 & 58 & 16 & 13 & 15 & 9 & 0 & 5 & 8 \\
\hline \multirow[t]{2}{*}{ L-Tryptophan } & 1 & 79 & 68 & 64 & 60 & 43 & 50 & 51 & 45 \\
\hline & 10 & 61 & 19 & 13 & 4 & 0 & 0 & 0 & 0 \\
\hline L-Tyrosine & 1 & 76 & 53 & 66 & 73 & 2 & 28 & 91 & 112 \\
\hline & 2 & 64 & 25 & 19 & 24 & 13 & 9 & 15 & 0 \\
\hline L-Valine & 1 & 107 & 94 & 91 & 84 & 90 & 98 & 95 & 54 \\
\hline & 10 & 66 & 59 & 74 & 78 & 18 & 104 & 111 & 67 \\
\hline Urea & 1 & 112 & 138 & 109 & 108 & 14 & 0 & 21 & 116 \\
\hline & 10 & 147 & 126 & 122 & 113 & 3 & 1 & 0 & 0 \\
\hline
\end{tabular}

activity was accompanied by only a slight reduction in growth, suggesting their ability to act as nitrogen sources at lower concentrations. Threonine did not show such a clear utilization by $\boldsymbol{A}$. cylindrica at $1 \mathrm{~mm}$ during the first $100 \mathrm{~h}$ of culture but there was evidence of its utilization as a nitrogen source between 100 and $200 \mathrm{~h}$ of culture.

Only two amino acids, cysteine (Fig. 4) and histidine, appeared to be toxic at both 1 and $10 \mathrm{~mm}$, suppressing growth and nitrogenase activity under buffered and unbuffered conditions. 


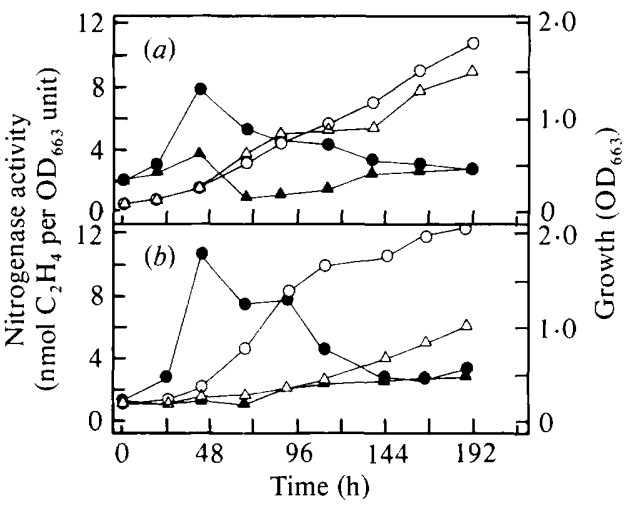

Fig. 3

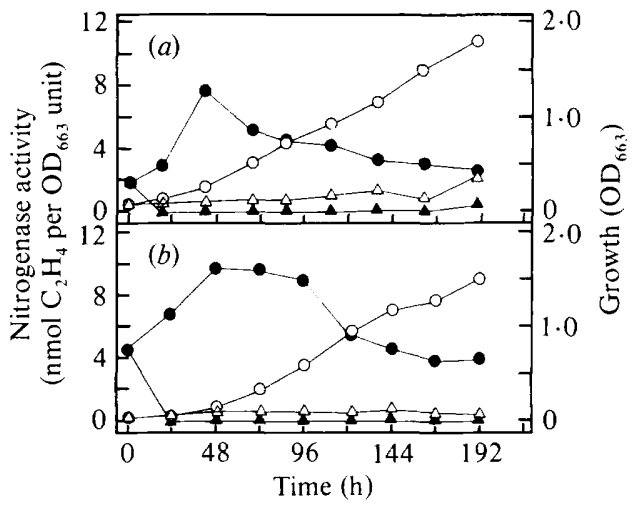

Fig. 4

Fig. 3. Nitrogenase activity $(\boldsymbol{\Delta}, \boldsymbol{O})$ and growth $(\triangle, O)$ of Anabaena cylindrica in control cultures ( O) and in cultures supplemented with $(a) 1$ mM-leucine $(\boldsymbol{\Delta}, \triangle)$ and $(b) 10$ mM-leucine $(\boldsymbol{\Delta}, \triangle)$. All cultures were buffered at $\mathrm{pH} 7.3$ with HEPES.

Fig. 4. Nitrogenase activity $(\boldsymbol{\Delta}, \boldsymbol{O})$ and growth $(\triangle, \bigcirc)$ of Anabaena cylindrica in control cultures $(\boldsymbol{O}$, $\bigcirc)$ and in cultures supplemented with $(a) 1$ mM-cysteine $(\boldsymbol{\Lambda}, \triangle)$ and $(b) 10$ mM-cysteine $(\boldsymbol{\Delta}, \triangle)$. All cultures were buffered at $\mathrm{pH} 7.3$ with HEPES.

\section{DISCUSSION}

Whilst exogenous glutamine has been shown previously to cause inhibition of nitrogenase in A. cylindrica (Rowell et al., 1977), it was thought that other major pool amino acids did not (Stewart, 1980). It is clear from the results described here that several amino acids have an effect on nitrogenase, with some being capable of acting as a source of nitrogen. The response of $\boldsymbol{A}$. cylindrica to individual exogenous amino acids was influenced by concentration, as seen in the variable effects at 1 and $10 \mathrm{~mm}$ in buffered cultures. Amino acid uptake is probably affected by $\mathrm{pH}$, and amino acid supplements to unbuffered cultures were shown to affect $\mathrm{pH}$. This makes comparison with control cultures difficult under unbuffered conditions, although with the majority of amino acids investigated similar patterns of response were seen in buffered and unbuffered conditions.

The results obtained with arginine, glutamate, methionine, phenylalanine and proline are very close to those obtained by Thomas et al. (1982) with Gloeocapsa sp., suggesting that nitrogenase inhibition by amino acids in different cyanobacteria may be less variable than was previously thought.

Amino acid toxicity in bacterial autotrophs has generally been attributed to imbalance of amino acid metabolism (Eccleston \& Kelly, 1972). It is particularly interesting to note that Kelly (1969) explained phenylalanine toxicity in Thiobacillus neapolitanus as due to phenylalanine acting as an end-point inhibitor of the first enzyme of the branched pathway for aromatic amino acid biosynthesis. Such an explanation may be applicable to cyanobacteria. Hall et al. (1982) showed that 3-deoxy-D-arabinoheptulosonate-7-phosphate (DAHP) synthase, the first step unique to the aromatic amino acid pathway, was inhibited by $55 \%$ in the presence of $0.5 \mathrm{~mm}^{-}$ phenylalanine in Anabaena 7122 . Inhibition by tyrosine was much less at $9 \%$, with no inhibition being caused by tryptophan. Also a $58 \%$ inhibition of shikimate/NADP dehydrogenase was caused by a mixture of phenylalanine, tyrosine and tryptophan, each at $0.5 \mathrm{~mm}$.

As reductions in DAHP synthase and shikimate dehydrogenase activities will result in reduced aromatic amino acid production, end-point inhibition may help explain the results reported here with tyrosine, tryptophan and phenylalanine. In all three cases inhibitory effects were seen only at high concentrations. 


\section{REFERENCES}

Allen, M. B. \& ARnon, D. I. (1955). Studies on nitrogen-fixing blue-green algae. I. Growth and nitrogen fixation by Anabaena cylindrica Lemm. Plant Physiology 30, 366-372.

BATT, T. \& BRown, D. H. (1974). The influence of inorganic nitrogen supply on amination and related reactions in the blue-green alga, Anabaena cylindrica Lemm. Planta 116, 27-37.

Chapman, J. S. \& Meeks, J. C. (1983). Glutamine and glutamate transport by Anabaena variabilis. Journal of Bacteriology 156, 122-129.

Eccleston, M. \& Kelly, D. P. (1972). Assimilation and toxicity of exogenous amino acids in the methane-oxidizing bacterium Methylococcus capsulatus. Journal of General Microbiology 71, 541554.

Gallon, J. R. (1980). Nitrogen fixation by photoautotrophs. In Nitrogen Fixation, pp. 197-238. Edited by W. D. P. Stewart \& J. R. Gallon. London: Academic Press.

Hall, G. C., Flick, M. B., Gherna, R. L. \& Jensen, R. A. (1982). Biochemical diversity for biosynthesis of aromatic amino acids among cyanobacteria. Journal of Bacteriology 149, 65-78.

Haystead, A., Dharmawardene, M. W. N. \& STEWART, W. D. P. (1973). Ammonia assimilation in a nitrogen fixing blue-green alga. Plant Science Letters 1, 439-445.

Kelly, D. P. (1969). Regulation of chemoautotrophic metabolism. III. DAHP synthase in Thiobacillus neapolitanus. Archiv für Mikrobiologie 69, 360369.

LEE-KADEN, J. \& Simonis, W. (1982). Amino acid uptake and energy coupling dependent on photosynthesis in Anacystis nidulans. Journal of Bacteriology 151, 229-236.

Neilson, A. H. \& Doudoroff, M. (1973). Ammonia assimilation in blue-green algae. Archiv für Mikrobiologie 89, 15-22.

Neilson, A. H. \& Larsson, T. (1980). The utilization of organic nitrogen for growth of algae: physiolorical aspects. Physiologia plantarum 48, 542-553.
Pearce, J., Leach, C. K. \& Carr, N. G. (1969). The incomplete tricarboxylic acid cycle in the blue-green alga Anabaena variabilis. Journal of General Microbiology 55, 371-378.

RippKa, R., Deruelles, J., Waterbury, J. B., Herdman, M. \& Stanier, R. Y. (1979). Generic assignments, strain histories and properties of pure cultures of cyanobacteria. Journal of General Microbiology 111, 1-61.

Rowell, P., Enticott, S. \& Stewart, W. D. P. (1977). Glutamine synthetase and nitrogenase activity in the blue-green alga Anabaena cylindrica. New Phytologist 79, 41-54.

Smith, G. D., Mackey, E. J. \& Daday, A. (1983). Buffer and salt damage to the filamentous cyanobacterium Anabaena cylindrica. Journal of General Microbiology 129, 3099-3102.

Stacey, G., Van Baalen, C. \& Tabita, F. R. (1979). Nitrogen and ammonia assimilation in the cyanobacteria: regulation of glutamine synthetase. Archives of Biochemistry and Biophysics 194, 457-467.

Stanier, R. Y. \& Cohen-Bazire, G. (1977). Phototrophic prokaryotes: the cyanobacteria. Annual Review of Microbiology 31, 225-274.

Stewart, W. D. P. (1980). Some aspects of structure and function in $\mathrm{N}_{2}$-fixing cyanobacteria. Annual Review of Microbiology 34, 497-536.

Stewart, W. D. P., Fitzgerald, G. P. \& Burris, R. H. (1967). In situ studies on $N_{2}$ fixation using the acetylene reduction technique. Proceedings of the National Academy of Sciences of the United States of America 58, 2071-2078.

Thomas, J. H., Mullineaux, P. M., Cronshaw, A. D., Chaplin, A. E. \& Gallon, J. R. (1982). The effect of structural analogues of amino acids on ammonia assimilation and acetylene reduction (nitrogen fixation) in Gloeocapsa (Gloeothece) sp. CCAP 1430/3. Journal of General Microbiology 128, 885-893.

VASHAMPaYAN, A. (1982). Amino acid nutrition in the blue-green alga Nostoc muscorum. New Phytologist 90, 545-549. 